\title{
EXACT PROPERTIES OF FROBENIUS NUMBERS AND FRACTION OF THE SYMMETRIC SEMIGROUPS IN THE WEAK LIMIT FOR $\mathrm{N}=3$
}

\author{
V. SHCHUR
}

\begin{abstract}
We generalize and prove a hypothesis by V. Arnold on the parity of Frobenius number. For the case of symmetric semigroups with three generators of Frobenius numbers we found an exact formula, which in a sense is the sum of two Sylvester's formulaes. We prove that the fraction of the symmetric semigroups is vanishing in the weak limit.
\end{abstract}

\section{Definitions}

Take $n$ mutually prime numbers $a_{1}, a_{2}, \ldots, a_{n}:\left(a_{1}, a_{2}, \ldots, a_{n}\right)=1$. Consider semigroup $S=\left\{s=x_{1} a_{1}+\ldots+x_{n} a_{n} \| x_{i} \in \mathbb{Z}_{+}\right\}$. 1 It follows that starting from some number $F\left(a_{1}, a_{2}, \ldots, a_{n}\right) \in S$ all integers are in the set $S$ :

Definition 1. $F\left(a_{1}, a_{2}, \ldots, a_{n}\right)=\min \left(s \in S \| \forall k \in \mathbb{Z}_{+}, k \geq s: k \in\right.$ $S)$.

Number $F\left(a_{1}, a_{2}, \ldots, a_{n}\right)$ is called a Frobenius number 2 and it's properties are the subject of the paper. For $n=2$ the exact formula is known, the Sylvester formula [1], for $F$ : $F\left(a_{1}, a_{2}\right)=\left(a_{1}-1\right)\left(a_{2}-1\right)$. It is known [2] that for an arbitrary relatively prime set $\left(a_{1}, a_{2}, \ldots, a_{n}\right)$, $n \geq 3$ the Frobenius number cannot be expressed in terms of a finite set of polynomials.

There are two useful functions. Function $C\left(a_{1}, a_{2}, \ldots, a_{n}\right)=F\left(a_{1}, a_{2}, \ldots, a_{n}\right)-$ 1 is the maximal integer which is not in the set $S$. Function $G\left(a_{1}, a_{2}, \ldots, a_{n}\right)=$ $F\left(a_{1}, a_{2}, \ldots, a_{n}\right)-1+a_{1}+a_{2}+\ldots+a_{n}$ is an analog of the function $C$ for the semigroup $S_{1}=\left\{s=x_{1} a_{1}+\ldots+x_{n} a_{n} \| x_{i} \in \mathbb{N}\right\}$.

Date: November 21, 2018.

1991 Mathematics Subject Classification. 2000 Math. Subject Classification : Primary 11P21; Secondary 11N56.

Key words and phrases. Frobenius numbers, semigroups, weak asymptotics.

${ }^{1}$ In the paper, $\mathbb{N}$ is the set of all positive integers, and $\mathbb{Z}_{+}$is the set of all non-negative integers.

${ }^{2}$ We have to note that often the Frobenius number defined as a number less by unity (compare with the function $C$ defined below). In the paper we will keep given notation which is also used in papers by V.Arnold. 
Note, that condition $s<F$ and $s \in S$ leads to the result that $C-s \notin S$ (otherwise, $C \in S$ ). The opposite statement is not true in the general case.

Definition 2. Semigroup $S$ is called symmetric if $\forall s<F, s \notin S$ : $C-s \in S$.

Each semigroup generated by two elements is the symmetric one.

\section{EXACT RESULTS}

Analyzing properties of Frobenius numbers and associated semigroups, V. Arnold noticed that if two of three generating elements are even then $F$ is even as well [3]. The following Theorem is the generalization of the experimental observation and gives the proof. Note, that renumbering of the generating elements does not change the structure of the set $S$ and, in particular, does not change value of $F$. For the sake of simplicity we shall use this property in following without explicitly mentioning it.

Theorem 1. Let us $\left(a_{1}, a_{2}, \ldots, a_{n-1}, a_{n}-1\right)=d$. Then $F$ is divisible by $d$.

Proof. By the definition of $F: F-1+a_{n} \in S\left(a_{n} \geq 1\right)$. Hence, there exist nonnegative integers $x_{1}, \ldots, x_{n}$ such that $F-1+a_{n}=x_{1} a_{1}+\ldots+$ $x_{n} a_{n}$. Therefore, $F-1=x_{1} a_{1}+\ldots+x_{n-1} a_{n-1}+\left(x_{n}-1\right) a_{n}$. If $x_{n} \geq 1$, then $F-1 \in S$, what contradicts to the definition of $F$. Hence, $x_{n}=0$ $F=x_{1} a_{1}+\ldots+x_{n-1} a_{n-1}-\left(a_{n}-1\right)$. Thus, $F$ is divisible by $d$.

Let $d_{i j}=\left(a_{i}, a_{j}\right), i \neq j$.

Lemma 1. Johnson's lemma [4]. $G\left(a_{1}, a_{2}, a_{3}\right)=d_{12} G\left(\frac{a_{1}}{d_{12}}, \frac{a_{2}}{d_{12}}, a_{3}\right)$.

Lemma 2. Brauer-Shockley lemma [5]. Let $d=\left(a_{1}, a_{2}, \ldots, a_{n-1}\right)$. Then, $C\left(a_{1}, a_{2}, \ldots, a_{n}\right)=d C\left(a_{1} / d, \ldots, a_{n-1} / d, a_{n}\right)+(d-1) a_{n}$.

Divisibility of $F$ by $d$ follows also 3 from the Lemma 2. Indeed, $F\left(a_{1}, \ldots, a_{n}\right)=C\left(a_{1}, \ldots, a_{n}\right)+1=d C\left(a_{1} / d, \ldots, a_{n-1} / d, a_{n}\right)+(d-1) a_{n}+$ $1=d\left(C\left(a_{1} / d, \ldots, a_{n-1} / d, a_{n}\right)+a_{n}\right)-\left(a_{n}-1\right)$.

Lemma 3. Semigroup $S\left(a_{1}, a_{2}, a_{3}\right)$ is symmetric if and only if for some $i: a_{i} \in S\left(\frac{a_{j}}{d_{j k}}, \frac{a_{k}}{d_{j k}}\right)$, where $i \neq j \neq k$.

In the case of symmetric semigroups we can prove the following exact formula for the Frobenius numbers with $n=3$. Denote $b_{l}=\frac{a_{l}}{d_{12}}, l=$ 1,2 . It is always possible to renumerate generating elements in such a way that under the conditions of Lemma 2 it takes place that $i=3$.

Theorem 2. Let $S\left(a_{1}, a_{2}, a_{3}\right)$ is a symmetric semigroup, $a_{3} \in$ $S\left(b_{1}, b_{2}\right)$. Then $F\left(a_{1}, a_{2}, a_{3}\right)=d_{12} F\left(\frac{a_{1}}{d_{12}}, \frac{a_{2}}{d_{12}}\right)+F\left(d_{12}, a_{3}\right)$.

\footnotetext{
${ }^{3}$ We acknowledge to referee for this remark.
} 
EXACT PROPERTIES OF FROBENIUS NUMBERS AND FRACTION OF THE SYMMETRIC SEMIGROUPS IN

Proof. $F\left(a_{1}, a_{2}, a_{3}\right)=G\left(a_{1}, a_{2}, a_{3}\right)-a_{1}-a_{2}-a_{3}+1=/$ by Lemma $1 /=d_{12} G\left(\frac{a_{1}}{d_{12}}, \frac{a_{2}}{d_{12}}, a_{3}\right)-a_{1}-a_{2}-a_{3}+1=d_{12}\left(F\left(b_{1}, b_{2}, a_{3}\right)+b_{1}+b_{2}+\right.$ $\left.a_{3}-1\right)-d_{12} b_{1}-d_{12} b_{2}-a_{3}+1=d_{12} F\left(b_{1}, b_{2}\right)+d_{12} a_{3}-a_{3}-d_{12}+1=/$ by Sylvester's formula $/=d_{12} F\left(b_{1}, b_{2}\right)+F\left(d_{12}, a_{3}\right)$. Note, that $\left(d_{12}, a_{3}\right)=1$ due to the $\left(a_{1}, a_{2}, a_{3}\right)=1$.

Notice. If semigroup $S\left(a_{1}, a_{2}, a_{3}\right)$ is not symmetric then $F\left(a_{1}, a_{2}, a_{3}\right)<$ $d_{12} F\left(\frac{a_{1}}{d_{12}}, \frac{a_{2}}{d_{12}}\right)+F\left(d_{12}, a_{3}\right)$, because if $a_{3} \notin S\left(b_{1}, b_{2}\right)$ then $F\left(b_{1}, b_{2}, a_{3}\right)<$ $F\left(b_{1}, b_{2}\right)$.

At the time of the paper revision the author found in the literature theorem in Chinese language [6] similar to our Theorem 2.

\section{AsYmptotic PROPERTIES}

Theorem 2 shows that, informally speaking, if $a_{3}$ is sufficiently large $\left(a_{3} \geq F\left(b_{1}, b_{2}\right)\right)$, than $F\left(a_{1}, a_{2}, a_{3}\right)$ behaves as a Frobenius number with two generating elements (compare with the Sylvester formula!). Therefore, it is interesting to consider the case under which all numbers $a_{1}, a_{2}, a_{3}$ are of the same order in some sense. V. Arnold [7] proposed to investigate an asymptotic behavior in the following way. Let us fix vector $\mathbf{a}=\left(a_{1}, \ldots, a_{n}\right)$ and for some function $f(\mathbf{a})$ consider the following average:

$$
V(f, \mathbf{a} r, N)=\sum_{-r \leq r_{i} \leq r, i=1, \ldots, n} f(N \mathbf{a}+\mathbf{r}) /(2 r)^{n}
$$

where $\mathbf{r}=\left(r_{1}, \ldots, r_{n}\right) \in \mathbb{Z}^{n}$ and $r, N \in \mathbb{N}$. Let us choose set $r(N)$ such that

$$
r(N) \rightarrow \infty, \frac{r(N)}{N} \rightarrow 0 \text { if } N \rightarrow \infty
$$

Let us consider the limit:

$$
L(f, \mathbf{a})=\lim _{r \rightarrow \infty, N \rightarrow \infty, r / N \rightarrow 0}(V) .
$$

Let $\operatorname{Sym}(\mathbf{a})=\left\{\begin{array}{rr}1 & \text { if } S\left(a_{1}, a_{2}, a_{3}\right)-\text { symmetric } \\ 0 & \text { - otherwise }\end{array}\right.$. Fel [8] gives theorem which in our terminology sounds as follows.

Theorem 3. $L(S y m, \mathbf{a})=0$.

Unfortunately, proof given in [8] contains some mistakes. For example, author says that set of triples $\left(a_{1} N+r_{1}, a_{2} N+r_{2}, a_{3} N+\right.$ $\left.r_{3}\right),\left|a_{i}\right| \leq r(N), i=1,2,3$, which constitute symmetric semigroups and such that $D=\left(a_{1} N+r_{1}, a_{2} N+r_{2}\right) \sim N$, is empty while $N \rightarrow \infty$. Let us give counter example. Let $a_{1}=4, r_{1}=2, a_{2}=6, r_{2}=3$. Then $D=2 N+1 \sim N$ and $F\left(\frac{a_{1} N+r_{1}}{D}, \frac{a_{2} N+r_{2}}{D}\right)=2$, i.e. for any 
set $r(N)$, fulfilling (11), and any $a_{3}$ for large enough $N$ semigroup $S\left(a_{1} N+r_{1}, a_{2} N+r_{2}, a_{3} N+r_{3}\right)$ (if exists) is symmetric. Thus, considered set are not always empty. We present here some other proof of the Theorem 3. The main idea is not to prove an emptiness but rather prove the fact that the fraction of symmetric semigroups is small in the considered limit.

We say that the fraction of the symmetric semigroups is vanishing in the weak asymptotics. Fel showed [8] that in the limit of interest the fraction of the mutually simple numbers (those sets on which Frobenius numbers defined) is equal $\frac{1}{\zeta(n)}$. In other words for

$$
I(\mathbf{a})=\left\{\begin{array}{rrr}
1 & \text { if } & \left(a_{1}, \ldots, a_{n}\right)=1 \\
0 & & \text { - otherwise }
\end{array} \text { we have } L(I, \mathbf{a}, 1)=\frac{1}{\zeta(n)} .\right.
$$

Proof. Let $A_{i}=N a_{i}+r_{i}$. Let us figure out the number of semigroups in the cubic $r$-vicinity of the point $N$ a those for which $A_{3} \in S_{0}=S\left(\frac{A_{1}}{D}, \frac{A_{2}}{D}\right)$, where $D=\left(A_{1}, A_{2}\right)$. Note, that for sufficiently large $D$ Frobenius number $F\left(\frac{A_{1}}{D}, \frac{A_{2}}{D}\right)$ is not larger then $A_{3}$, therefore for sufficiently large $D$ any $A_{3}$ lies in semigroup $S_{0}$. The number of semigroups such that $D \geq D_{0}$ (value of $D_{0}$ will be chosen later on) may be estimated as:

$$
U_{1}=\sum_{D=D_{0}}^{c N}\left(\frac{2 r}{D}\right)^{2} \leq 4 r^{2} \int_{D_{0}-1}^{c N-1} \frac{1}{x^{2}} d x=4 r^{2}\left(\frac{1}{D_{0}-1}-\frac{1}{c N}\right)
$$

where constant $c$ may be chosen as $c=\min \left(a_{1}, a_{2}\right)+1$ because if $N \rightarrow \infty, r \rightarrow \infty, r / N \rightarrow 0$ then $D \leq \min \left(A_{1}, A_{2}\right) \leq c N$.

Now consider the case $D<D_{0}$. Let us fix some $\varepsilon>0$. Then $\exists N_{0}$ such that $\forall N>N_{0}$ we have $r(N)<\varepsilon N$, i.e. $\forall N>N_{0} \forall r_{3}$ : $\left|r_{3}\right|<r(N)$ we have that $a_{3} N+r_{3}<\left(a_{3}+\varepsilon\right) N$. Denote $c_{1}=a_{3}+\varepsilon$. Let us estimate the number of elements of the semigroup $S\left(\frac{A_{1}}{D}, \frac{A_{2}}{D}\right)$ in the interval $\left[0 ; c_{1} N\right]: s=x_{1} a_{1}+x_{2}+a_{2}$. (See Fig. 1) Without lack of generality let that $a_{1}<a_{2}$. Then for sufficiently large $N \frac{A_{1}}{D}<\frac{A_{2}}{D}$. Denote $T=\left[\frac{c_{1} N D}{A_{1}}\right]$. Notice that $x_{1}+x_{2} \leq T$. With fixed number $x_{2}$ number $x_{1}$ may take not more than $T-x_{2}$ values. Therefore,

$$
\text { \# }\left(S\left(\frac{A_{1}}{D}, \frac{A_{2}}{D}\right) \bigcap\left[0 ; c_{1} N\right]\right) \leq \sum_{x_{2}=0}^{T}\left(T-x_{2}\right)=\sum_{k=0}^{T} k=\frac{T(T+1)}{2}
$$

Then with fixed $A_{1}$ and $A_{2}$ and any $A_{3}=a_{3} N+r_{3},\left|r_{3}\right| \leq r(N)$ the number of symmetric semigroups are not larger than $\left(S\left(\frac{A_{1}}{D}, \frac{A_{2}}{D}\right) \cap\left[0 ; c_{1} N\right]\right)$. So the number of symmetric semigroups such that $D<D_{0}$, may be 
estimated as

$$
U_{2} \leq \sum_{D=1}^{D_{0}}\left(\frac{2 r}{D}\right)^{2}\left(C_{1}^{2}\left(\frac{N}{A_{1}}\right)^{2} D^{2}+O(D)\right) \approx 4 r^{2} C_{1}^{2}\left(\frac{N}{a_{1} N+r_{1}}\right)^{2} D_{0} .
$$

Thus, the fraction of symmetric semigroups is estimated as

$$
W=3 \frac{U_{1}+U_{2}}{8 r^{3}} \leq 3\left(\frac{1}{D_{0}-1}-\frac{1}{c N}+\frac{D_{0}}{2 r} C_{1}^{2}\left(\frac{N}{a_{1} N+r_{1}}\right)^{2}\right) .
$$

Let $D_{0}=\ln r$. Then, with $N \rightarrow \infty, r \rightarrow \infty, r / N \rightarrow 0$ the right hand side of inequality (2) vanishes, i.e. $W \rightarrow 0$.

Acknowledgment. Author acknowledge scientific advises by V.I. Arnold and S.M. Gusein-Zade and help from A. Ustinov. This work supported by the Russian Federation Grant NSh-709.2008.1.

\section{REFERENCES}

[1] J.J. Sylvester, Educational Times 41 (1884) 21.

[2] F. Curtis, Math. Scand. 67 (1990), 190.

[3] V.I. Arnold, seminar talk, Moscow State University, 04.10.2005.

[4] S.M. Johnson, Canad. J. Math. 12 (1960) 390.

[5] A. Brauer and J.E. Shockley, J.Reine Angewandte Math. 211 (1962), 215.

[6] C. Niu and Z. Oiu, (Chinese) J. Shandong Univ. Nat. Sci. Ed. 21 (1986) 1.

[7] V.I. Arnold, Mathematical Physics, Analysis and Geometry 9 (2006) 95.

[8] L.G. Fel, Func. Anal. and Other Math., doi:10.1007/s11853-008-0019-y 


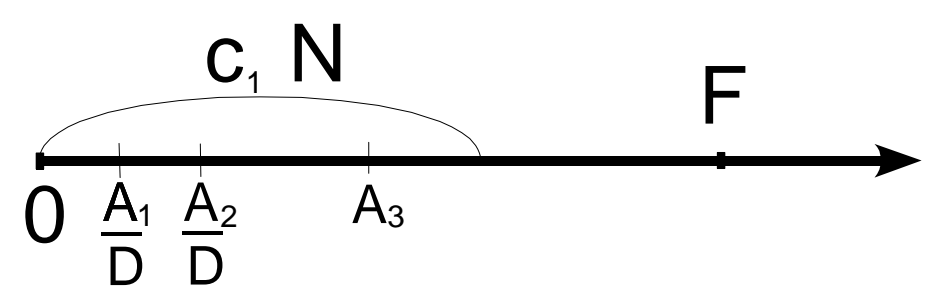

Figure 1.

Faculty of Mechanics and Mathematics, Lomonosov Moscow State University,GSP-2, Leninskie Gory, Moscow, 119992, Russian FederaTION

E-mail address: vladimir@chg.ru 